\title{
Clinical Profile of Patients with Systemic Lupus Erythematosus
}

\author{
Paudyal BP,' Gyawalee $M^{2}$ \\ 'Department of Internal Medicine, ${ }^{2}$ Department of Dermatology, Patan Academy of Health Sciences, Patan Hospital.
}

\section{ABSTRACT}

Introduction: Clinical features of Systemic lupus erythematosus vary between different parts of the World; however, this information on Nepalese Sytemic lupus erythematosus does not exist.

Methods: Patients with Systemic lupus erythematosus fulfilling the American College of Rheumatology criteria treated in Patan Hospital were studied by means of retrospective review of their case records. The results were compared with various regional and international studies.

Results: The female to male ratio was 36:1 with a mean age at diagnosis 28.67 years. Cutaneous manifestations were the most common 72 (96\%) presentations followed by musculoskeletal involvement 70 (93\%). Haematological and renal involvements each were present in $33(44 \%)$ of patients. Pulmonary and neuropsychiatric involvements were present in $15(20 \%)$ and 8 $(11 \%)$ patients respectively. Antinuclear antibody and anti-double stranded deoxyribonucleic acid antibodies were positive in $70(93 \%)$ and $66(88 \%)$ of patients respectively. A quarter of patients had some form of co-morbid conditions. At the time of last follow up, 70 (93\%), 66 $(90 \%)$, and $6(9 \%)$ of patients were taking antimalarials, steroids and immunosuppressive agents respectively, mostly in combinations. Eighty seven percent of patients had their disease activity under control at the time of last follow up whereas four percent still had an active disease. In another four percent died during the course of therapy and the outcome was unknown in another four percent of patients who lost to follow up.

Conclusions: Clinical characteristics and antinuclear antibody pattern of Nepalese lupus patients is more or less similar to those with patients from other countries in the region and in the World.

Keywords: clinical profile; Nepal; systemic lupus erythematosus.

\section{INTRODUCTION}

Systemic lupus erythematosus (SLE) is a chronic multi system autoimmune disorder mainly affecting young women. ${ }^{1}$ While there has been a significant improvement in the survival of these patients over the past four decades, the incidence of SLE has nearly tripled in the same period. ${ }^{2}$

The development of SLE is dependent on environmental and genetic factors, though, the mechanisms behind clinical findings and etiologic events remain largely unclear. ${ }^{3}$ Prevalence rates and predominant symptoms differ in different ethnic populations. ${ }^{4}$ Several studies have shown that SLE is more common among Asians when compared to Caucasians. SLE was three times

Correspondence: Dr. Buddhi P Paudyal, Department of Internal Medicin, Patan Academy of Health Sciences, Patan Hospital, Lagankhel, Lalitpur, Nepal. Email: buddhipaudyal@yahoo.com, Phone: 977-15522266 . 
more common among people of South Asian origin than Caucasians who reside in Leicester city, England. ${ }^{5}$ Similarly, Serdula and Rhoads' survey of SLE patients in Hawaii showed the age-adjusted prevalence rates for SLE were significantly higher in Eastern Asians than that in Caucasians. ${ }^{6}$ Moreover, regional differences in the clinical patterns and causes of death in SLE have been described in many countries.

The incidence and prevalence of SLE in many developing countries including Nepal is unknown. Similarly, clinical, mortality and serological data on SLE is sparse. In this context, an attempt has been made to investigate the clinical profile, serological characteristics, and prognosis of Nepalese SLE patients from Patan hospital.

\section{METHODS}

This is a retrospective cross-sectional study of SLE patients fulfilling the American College of Rheumatology criteria $^{7}$ treated in the Department of Medicine, Patan Hospital. The data were collected between May to July 2011. Inclusion criteria comprised of patients who fulfilled at least four of the American College of Rheumatology (ACR) criteria for the classification of disease revised in 1997 and had their regular visit in the Patan Hospital from September 2008 to April 2011. Ethical approval was taken from institutional review board of Patan Hospital.

Patan Hospital is a tertiary level teaching hospital located in the Kathmandu valley and serves people from the valley and also as a referral center for the rest of the country. It provides services to approximately 2,50,000 outpatients and 22000 inpatients in a year. Owing to the availability of specialist service, patients with rheumatic disease from different parts of the country are referred to this centre.

The clinical and laboratory manifestations meeting the ACR criteria were: 1) malar rash; 2) discoid rash; 3) photosensitivity; 4) oral ulcers; 5) arthritis; 6) serositis including pleuritis and pericarditis; 7) renal disorder; 8) neurological disorder; 9) hematological disorder including hemolytic anemia, leucopenia, lymphopenia and thrombocytopenia; 10) immunological disorder including abnormal anti double-stranded deoxyribonucleic acid (ds-DNA) titer, presence of antibody to Smith nuclear antigen (anti-Sm) and positive finding of antiphospholipid antibodies; and 11) abnormal titer of antinuclear antibody (ANA).

The laboratory data collected included a standard hematological and immunologic profile (complete blood count, erythrocyte sedimentation rate, serum electrolytes, serum creatinine, 24 hour urinary protein and creatinine clearance and various immunological tests including ANA, anti-ds DNA, and antiphospholipid antibodies and serum complements levels in selected cases). Where indicated, imaging studies and other invasive procedures (including biopsies) were performed. The current status of disease at the time of data collection and the relative incidence of the various complications that ultimately resulted in morbidity and mortality were noted. Presence of any other comorbid conditions was recorded. Drug therapy at the time of last follow up was also documented. Statistical package for social science (SPSS) (Release 10.0.5, standard version, 1989-99) was used for data analysis. The results were compared with various regional and international studies.

\section{RESULTS}

A total of 75 SLE patients were studied. Of these, 73 were females and 2 were males. The mean age of the patients was 33.03 years with a range of $8-75$ years. The mean age at diagnosis was 28.67 years with a range of 6-74 years. The mean duration of disease was 4.36 years with a range of 1-17 years.

Cutaneous manifestations were the most common presentation 72 (96\%) in our series. Malar rash and photosensitivity were present in 51 (69\%) and 29 $(39 \%)$ of cases whereas alopecia and discoid rashes manifested in less than one third of cases only. Musculoskeletal involvement was the second most common 69 (93\%) organ/ system involvement in our patients. Patients with musculoskeletal involvement presented either with arthralgia or arthritis; we did not find a single case of clinical muscle involvement. Two patients ( $3 \%$ ) developed avascular necrosis (AVN) of femoral heads; both of them developed bilateral involvement during the course of time. Oral ulcer was the next most frequent manifestation, seen in $45(60 \%)$ of our SLE patients (Table 1).

Haematological and renal involvements each were present in $33(44 \%)$ of our patients. The most common haematological involvement was anaemia followed by thrombocytopenia and leucopenia. Of those with renal involvement, all presented with some degree of proteinuria whereas haematuria was seen less commonly. Five patients $(7 \%$ of total patients and $15 \%$ of those with renal involvement ) had renal biopsy: the biopsy revealed World Health Organization (WHO) class III lesion (focal proliferative glomerulonephritis) in one, WHO class IV lesion (diffuse proliferative glomerulonephritis) in three, and WHO class $\mathrm{V}$ lesion (membraneous glomerulonephritis) in one case. 
Table 1. The cumulative prevalence of organ/ system involvement and clinical manifestations of lupus patients.*

\begin{tabular}{|c|c|}
\hline Clinical features & n (\%) \\
\hline Cutaneous & $72(96 \%)$ \\
\hline Malar rash & $51(69 \%)$ \\
\hline Photosensitivity & $29(39 \%)$ \\
\hline Alopecia & $24(32 \%)$ \\
\hline Discoid rash & $17(23 \%)$ \\
\hline Musculoskeletal & $69(93 \%)$ \\
\hline Arthritis/arthralgia & $69(93 \%)$ \\
\hline Avascular necrosis & $2(3 \%)$ \\
\hline Gastrointestinal & $45(60 \%)$ \\
\hline Oral lesions & $45(60 \%)$ \\
\hline Haematological & $33(44 \%)$ \\
\hline Anaemia & $21(28 \%)$ \\
\hline Thrombocytopenia & $6(8 \%)$ \\
\hline Leucopenia & $5(7 \%)$ \\
\hline Autoimmune haemolytic anaemia & $1(1 \%)$ \\
\hline Renal & $33(44 \%)$ \\
\hline Proteinuria & $33(44 \%)$ \\
\hline Haematuria & $17(23 \%)$ \\
\hline Biopsy proven Nephritis & $5(7 \%)$ \\
\hline Pulmonary & $15(20 \%)$ \\
\hline Pleurisy & $12(16 \%)$ \\
\hline Lupus pneumonitis & $2(3 \%)$ \\
\hline Interstitial Lung Disease & $2(3 \%)$ \\
\hline Pulmonary hypertension & $1(1 \%)$ \\
\hline Cardiovascular & $9(13 \%)$ \\
\hline Vasculitis & $5(7 \%)$ \\
\hline Thrombosis & $3(4 \%)$ \\
\hline Pericardial effusion & $1(1 \%)$ \\
\hline Myocarditis & $1(1 \%)$ \\
\hline Raynaud's phenomenon & $9(12 \%)$ \\
\hline Neuropsychiatric & $8(11 \%)$ \\
\hline Seizure & $5(7 \%)$ \\
\hline Confusion & $1(1 \%)$ \\
\hline Peripheral neuropathy & $1(1 \%)$ \\
\hline Headache & $1(1 \%)$ \\
\hline Fever & $39(53 \%)$ \\
\hline Eye & $1(1 \%)$ \\
\hline Retinal vasculitis & $1(1 \%)$ \\
\hline
\end{tabular}

* Some of the patients had more than one features 
Pulmonary involvement was present in 15 (20\%) of our patients with SLE. The most frequent pulmonary involvement was pleurisy followed by lupus pneumonitis and interstitial lung disease (ILD); one patient with ILD developed pulmonary hypertension. Cardiovascular involvement was seen in $9(13 \%)$ patients. Five patients (7\%) had vasculitis (mostly cutaneous vasculitis) whereas three developed thrombosis. Pericarditis and myocarditis was seen in one patient each; the patient with later condition died during the course of treatment.

Neuropsychiatric involvement was seen in 8 (11\%) patients. Seizure was the most frequent presentation $7 \%$ followed by confusion, neuropathy and headache ( $1 \%$ each). One patient had significant eye involvement in the form of retinal vasculitis. More than half $(53 \%)$ of our patients had fever, mainly during the time of initial presentation. Only $9(12 \%)$ of these patients had Raynaud's phenomenon sometime during the course of illness.

In the serology, ANA was positive in 69 (93\%) and negative in $3(5 \%)$ patients. Anti ds- DNA antibody was present in $66(88 \%)$ of patients. Both ANA and ds-DNA was missing in one patient. A clinical and laboratory criteria for confirmed antiphospholipid syndrome (APS) were met in $4(6 \%)$ of our patients.

A quarter of our patients (25\%) had some form of comorbid conditions. The common co-morbidities included hypothyroidism in $8(11 \%)$, hypertension in $6(8 \%)$, diabetes mellitus in $3(4 \%)$ and ischaemic heart disease in $1(1 \%)$, with some patients having more than one co-morbidities.

At the time of last follow up, 69 (93\%), 67 (90\%), and $5(9 \%)$ of our patients were taking antimalarials, steroids and immunosuppressive agents respectively, mostly in the combination of two or more.

At the time of last follow up, majority $(88 \%)$ of our patients had their disease activity under control whereas $3(4 \%)$ still had an active disease (proteinuria in two, cytopenia and pneumonitis in one each). Three patients (4\%) died during the course of therapy. The causes of death were sepsis in the background of severe lupus nephritis in one patient, mesenteric vasculitis in one, and lupus myocarditis in one patient. The outcome is unknown in another three patients (4\%) who lost to follow up (Table 2).

Table 2. Outcome of SLE patients.

\begin{tabular}{lc}
\hline Outcome & Frequencies \\
\hline Disease under control & $66(88 \%)$ \\
\hline Active lupus & $3(4 \%)$ \\
\hline Death & $3(4 \%)$ \\
Unknown (lost follow up) & $3(4 \%)$ \\
\hline
\end{tabular}

\section{DISCUSSION}

In this study we describe the clinical and serological profile, disease outcome, and treatment pattern of 75 Nepalese patients with SLE treated in a tertiary referral centre. The comparison of clinical manifestations and ANA profile of Nepalese and other lupus patients has been presented (Table 3). 
Paudyal et al. Clinical Profile of Patients with Systemic Lupus Erythematosus.

Table 3. Comparison of clinical manifestations and ANA profile of SLE patients between Nepalese and other populations.

\begin{tabular}{|c|c|c|c|c|c|c|c|}
\hline & Nepalese & Indian 9 & Pakistani $^{3}$ & $\begin{array}{l}\text { Hong Kong } \\
\text { Chinese }^{10}\end{array}$ & European ${ }^{11}$ & Tunisian $^{12}$ & $\begin{array}{l}\text { Puerto } \\
\text { Rico }^{13}\end{array}$ \\
\hline Number of patients & 75 & 1366 & 196 & 709 & 1000 & 100 & 134 \\
\hline $\mathrm{F} / \mathrm{M}$ & $73 / 2$ & -- & $172 / 24$ & -- & $908 / 92$ & $92 / 8$ & $127 / 7$ \\
\hline $\begin{array}{l}\text { Mean/ Median age (yr) at } \\
\text { diagnosis }\end{array}$ & 28.67 & $24-25$ & 31 & 30.1 & 29 & 32 & 32 \\
\hline Photosensitivity (\%) & 39 & 48 & 6 & 35 & 18.7 & 53 & 76.9 \\
\hline Malar rash (\%) & 69 & 58.5 & 29 & 56 & 26.4 & 63 & 56 \\
\hline Discoid rash (\%) & 23 & 7 & 14 & 12 & 5.4 & 18 & 10.4 \\
\hline Oral ulcers (\%) & 60 & 58.5 & 19.7 & 11 & 8.9 & 4 & 29.9 \\
\hline Arthropathy (\%) & 93 & 85 & 38 & 84 & 41.3 & 78 & 67.2 \\
\hline Serositis (\%) & 17 & 22 & 22 & 19 & 12.9 & 45 & 27.6 \\
\hline Renal (\%) & 44 & 73 & 33 & 50 & 22.2 & 43 & 29.9 \\
\hline Neuropsychiatric (\%) & 11 & 51 & 26 & 5 & 13.6 & 25 & 9 \\
\hline Haematologic (\%) & 44 & -- & 54 & -- & 12.8 & 81 & 76.9 \\
\hline ANA (\%) & 93 & -- & 86 & -- & 96 & 100 & 93.3 \\
\hline
\end{tabular}

Demographically, SLE was seen predominantly in females with a female to male ratio of $36: 1$. Most of the Asian studies ${ }^{8}$ have reported female to male ratio ranging from $7: 1$ to $28: 1$; the very high female to male ratio in our study could be due to small sample size with less male patient enrollment, or it is also possible that Nepali males are genetically less susceptible to develop SLE. Though there were only two male patients, both of them had major organ involvement (pericardial effusion with ischaemic necrosis of digits in one and WHO class IV glomerulonephritis in another).The mean age at diagnosis of our patients was during third decade of life, which was consistent with data from other cohorts including Indian, ${ }^{9}$ Chinese, $^{10}$ Arabs $^{4}{ }^{4}$ and European Caucasians. ${ }^{11}$

In general, cutaneous, musculoskeletal, oral mucosal, haematological, and renal manifestations were more frequent than pulmonary, cardiovascular, neuropsychiatric, and eye involvement in our lupus population. In the cutaneous manifestations, malar rash was much more common than photosensitivity, alopecia and discoid rashes which were seen in less than half of patients. These findings are comparable to the findings from other series who have noticed similar high prevalence of malar rashes. ${ }^{9,12,14}$ Discoid rashes were higher in Nepalese SLE patients, though there is high degree of variability in its occurrence. ${ }^{11,12}$ All of the patients with musculoskeletal involvement had either arthralgia or arthritis, clinical muscle involvement was, however, not seen in this patient population. Two patients developed avascular necrosis of femoral heads; both of them developed bilateral involvement during the course of time.

We did not observe gastrointestinal manifestations other than oral lesions in this patient population. One patient with severe lupus developed mesenteric vasculitis during a hospital admission and later succumbed to it. Common haematological manifestations in Nepalese SLE patients were anaemia followed by thrombocytopenia, and leucopenia; autoimmune haemolytic anaemia and lymphopenia were not remarkable in our cohort. This is in contrast to other studies from Asia and Europe who have observed lymphopenia in $20-70 \%$ of the patients with SLE. ${ }^{15,16}$

Renal involvement in lupus poses a major threat to morbidity and mortality, particularly among patients of Hispanic, and African-American ethnicity. ${ }^{1}$ Generally, renal involvement is more commonly encountered in blacks, Indians, and Chinese, with lesser prevalence in Caucasians, Puerto Rico and Arabs. ${ }^{3}$ Almost half (44\%) of our patients had lupus renal involvement which is similar to study from Arab, ${ }^{12,17}$ Europe, ${ }^{11,18}$ South Africa ${ }^{1}$ and Hong Kong Chinese ${ }^{10}$ population. Though only $15 \%$ of our patients with renal involvement had renal biopsy, the presence of more severe forms of lupus nephritis in renal biopsy suggests that only selected cases with severe disease were subjected to this procedure. Other factors for low renal biopsy rate could be due to patient denial, and most importantly unavailability of routine renal biopsy service in our hospital during the period of study. 
Serositis, an important feature of SLE, was seen less commonly $(17 \%)$ in our lupus patients. Pleurisy was observed in $12(16 \%)$ of patients whereas pericardial effusion was seen in only one patient. This could be due to low index of suspicion to these clinical features or a real low incidence in our population as in Australian Caucasian and Aborigines. ${ }^{19}$

Pulmonary, cardiovascular, and neuropsychiatric involvements were seen in less than one-fifth of our patients. These systemic involvements are usually associated poorer prognosis in SLE patients. The frequency is in line with other Asian cohorts ${ }^{8}$ except that Malaviya found higher rate $60 \%$ (45) of neuropsychiatric manifestations in Indian population. ${ }^{9}$ One of our patients had significant eye involvement due to retinal vasculitis.

ANA and ds-DNA were positive in 69 (93\%) and 66 $(88 \%)$ respectively. The prevalence of ANA in our lupus patients is similar to other SLE populations from different parts of the World.8,9,17 Definite antiphospholipid syndrome was observed in $6 \%$ (4) of our patients; this seems to be lower than other studies ${ }^{12}$ which have described higher prevalence of this syndrome in lupus patients. Prevalence of other antibodies like anti-Sm is not the part of the study as these are not routinely done.

Our lupus patients had significant comorbid conditions; at the mean age of diagnosis of 33 years, almost one fourth had developed some co-morbidity. Hypothyroidism in our SLE patients is most likely autoimmune in nature, though we do not test anti-microsomal antibodies routinely. The increased prevalence of hypertension, diabetes mellitus, and ischaemic heart disease in SLE patients can be explained as part of heightened cardiovascular risk owing to underlying inflammatory condition of lupus itself as well as due to the sequel of its treatment, ${ }^{20}$ as $67(90 \%)$ of these patients were taking steroids at the time of last follow up.

At the time of last follow up majority of the patients $66(88 \%)$ were enjoying low disease activity whereas $4 \%$ still had an active disease. Three patients (4\%) died during the course of therapy. All deaths occurred during the first five years of disease onset.We did not observe late-onset deaths or bimodal patterns of causes of death ${ }^{21}$ as seen in industrialized countries, probably because of small sample size and shorter follow up period. Sepsis and increased lupus activity were the main cause of death in our patients which is in parallel with the data from other studies. ${ }^{3,12}$ Non compliance and loss during follow up was a problem, though not as high as in other studies which have reported up to $28 \%$ of loss during follow up. ${ }^{1}$

Our study has several limitations. Though, being one of the initial studies of Nepalese lupus patients, it has many shortcomings of retrospective design. Being a descriptive study, it can not give statistical associations. Small sample size and data collected from one centre may not represent the lupus population of the entire country. Notwithstanding these limitations, we believe that our findings give a bird's eye view of lupus scenario in Nepal. Comprehensive lupus database and prospective studies are needed to have an idea on the clinical, serological, and prognostic profile of Nepalese lupus patients.

\section{ACKNOWLEDGEMENTS}

The authors would like to thank Dr. Buddha Basnyat for his constant support and help during the preparation of this manuscript. Similarly, we would like to thank Anil Sharma and Macha Bhai Shakya for their help in the computer work.

\section{REFERENCES}

1. Wadee S, Tikly M, Hopley M. Causes and predictors of death in South Africans with systemic lupus erythematosus. Rheumatology (Oxford). 2007 Sep;46(9):1487-91.

2. Uramoto KM, Michet CJ Jr, Thumboo J, Sunku J, O'Fallon WM, Gabriel SE. Trends in the incidence and mortality of systemic lupus erythematosus, 1950-1992. Arthritis Rheum. 1999 Jan;42(1):46-50.

3. Rabbani MA, Siddiqui BK, Tahir MH, Ahmad B, Shamim A, Shah SMA and Ahmad A. Systemic Lupus Erythematosus in Pakistan. Lupus. 2004;13:820-5.
4. Heller T, Ahmed M, Siddiqqi A, Wallrauch C and Bahlas S. Systemic lupus erythematosus in Saudi Arabia: morbidity and mortality in a multiethnic population. Lupus. 2007;16:908-15

5. Serdula MK, Rhoads GG. Frequency of systemic lupus erythematosus in different ethnic groups in Hawaii. Arthritis Rheum. 1979;22:328-33.

6. Samanta A, Roy S, Feehally J, Symmons DP. The prevalence of diagnosed systemic lupus erythematosus in Whites and Indian Asian immigrants in Leicester city, UK. Br J Rheumatol. 1992;31:679-82. 
7. Smith EL, Rhmerling S. The American College of Rheumatology criteria for the classification of systemic lupus erythematosus. Strengths, weaknesses and opportunities for improvement. Lupus. 1999;8:586-95.

8. Navarra SV and King JO. An overview of clinical manifestations and survival of systemic lupus erythematosus patients in Asia. APLAR Journal of Rheumatology. 2006;9:336-41.

9. Malaviya AN, Chandrasekaran AN, Kumar A, Sharma PN. Systemic lupus erythematosus in India. Lupus. 1997;6:690-700.

10. Mok CC, Lau CS. Lupus in Hong Kong Chinese. Lupus. 2003;12:717-22.

11. Cervera R, Khamashta MA, Font J, et al. Systemic lupus erythematosus: clinical and immunologic patterns of disease expression in a cohort of 1000 patients. The European Working Party on Systemic Lupus Erythematosus. Medicine (Baltimore). 1993;72:113-24.

12. Houman MH, Smiti-Khanfir M, Ben Ghorbell I and M Miled. Systemic lupus erythematosus in Tunisia: demographic and clinical analysis of 100 patients. Lupus. 2004;13:204-11.

13. Vila LM, Mayor AM, Valenti AH, Garcı Sobera M, Vila S. Clinical and immunological manifestations in 134 Puerto Rican patients with systemic lupus erythematosus. Lupus. 1999;8:279-86.
14. Thumboo J, Fong KY, Chng HH, et al. The effects of ethnicity on disease patterns in 472 orientals with systemic lupus erythematosus. J Rheumatol. 1998;25:1299-1304.

15. Alballa SR. Systemic lupus erythematosus in Saudi patients. Clin Rheumatol. 1995;14:342-6.

16. Nossent JC, Swaak AJ. Prevalence and significance of haematological abnormalities in patients with systemic lupus erythematosus. Q J Med. 1991;80:605-12.

17. Al-Jarallah K, Al-Awadi A, Siddiqui H, et al. Systemic lupus erythematosus in Kuwait-hospital based study. Lupus. 1998;7:434-8.

18. Blanco FJ, De la Mata J, Gomez-Reino JJ, et al. Manifestacionesclinicas y serologicas de 307 pacientesespanfoles con lupus eritematososistemico.Comparacion con otrosgruposetnicos. Rev Clin Exp. 1995;195:534-40.

19. Segasothy M, Phillips PA. Systemic lupus erythematosus in Aborigines and Caucasians in central Australia: a comparative study. Lupus. 2001;10:439-44.

20. Manzi S and Wasko MC. Inflammation- mediated rheumatic diseases and atherosclerosis. Annals of Rheumatic Diseases. 2000;59:321-5.

21. Urowitz MB, Bookman AAM, Koehler BE, Gordan DA, Smythe HA, Ogryzlo MA. The bimodal mortality pattern of systemic lupus erythematosus. Am J Med. 1976; 60:221-5. 